OPEN ACCESS

Edited by:

Crochan John O'Sullivan,

Triemli Hospital, Switzerland

Reviewed by:

Konstantinos Stathogiannis,

Stanford University, United States

Cameron Dowling,

Monash Health, Australia

${ }^{*}$ Correspondence:

Albert Markus Kasel

markus.kasel@usz.ch

Specialty section:

This article was submitted to Structural Interventional Cardiology,

a section of the journal

Frontiers in Cardiovascular Medicine

Received: 10 September 2021 Accepted: 11 October 2021

Published: 02 November 2021

Citation:

Chen M, Stähli BE, Michel JM Brinkert M, Tanner FC and Kasel AM

(2021) Case Report: Sapien 3

Transcatheter Heart Valve Embolization: Cause, Management, and Redo.

Front. Cardiovasc. Med. 8:774047.

doi: 10.3389/fcvm.2021.774047

\section{Case Report: Sapien 3 Transcatheter Heart Valve Embolization: Cause, Management, and Redo}

\author{
Mi Chen ${ }^{1,2}$, Barbara E. Stähli ${ }^{1}$, Jonathan M. Michel ${ }^{1}$, Miriam Brinkert ${ }^{3}$, Felix C. Tanner ${ }^{1}$ \\ and Albert Markus Kasel ${ }^{1 *}$ \\ ${ }^{1}$ University Heart Center, University Hospital Zurich, Zurich, Switzerland, ${ }^{2}$ Department of Cardiac Surgery, Beijing Anzhen \\ Hospital, Capital Medical University, Beijing, China, ${ }^{3}$ Department of Cardiology, Kantonsspital Aarau, Aarau, Switzerland
}

The transcatheter heart valve (THV) embolization is a rare but challenging complication in transcatheter aortic valve implantation (TAVI). We report the case of an 81-year-old man with Sapien 3 embolization caused by interrupted rapid pacing. In this setting, we describe the embolized THV management and the technique of the second Sapien 3 implantation.

Keywords: TAVI - transcatheter aortic valve implantation, embolization, transcatheter heart valve (THV), Sapien 3, aortic stenosis

\section{PATIENT HISTORY}

An 81-year-old man with hypertension, permanent atrial fibrillation, and chronic renal disease presented to the cardiology department complaining of syncope and dyspnea. Transthoracic echocardiography (TTE) showed severe paradoxical low-flow, low-gradient aortic stenosis of a tricuspid aortic valve (mean transaortic pressure gradient $=33 \mathrm{mmHg}$; aortic valve area $=0.96$ $\mathrm{cm}^{2}$; indexed aortic valve area $=0.49 \mathrm{~cm}^{2} / \mathrm{m}^{2}$; aortic valve $\mathrm{V}_{\max }=3.66 \mathrm{~m} / \mathrm{s}$; left ventricular ejection fraction $=63 \%$ ) (1). Pre-transcatheter aortic valve implantation (TAVI) computed tomography (CT) demonstrated a large aortic annulus with only minor calcifications (annulus area $=685 \mathrm{~mm}^{2}$, area-derived diameter $=29.53 \mathrm{~mm}$ ). Coronary angiography showed insignificant coronary artery stenosis.

\section{MANAGEMENT}

\section{Embolization of Sapien 3 THV}

The Heart Team decided to proceed with transfemoral TAVI given the patient's age, frailty, and the burden of comorbidities. Based on the annulus dimensions, TAVI with a 29-mm Sapien 3 (Edwards Lifesciences, Irvine, California) balloon-expandable transcatheter heart valve (THV) with $2 \mathrm{ml}$ overfilling was planned.

Transcatheter aortic valve implantation was performed under conscious sedation in the hybrid operating room. Having obtained vascular access, the THV was advanced and positioned within the aortic annulus. After rapid pacing had started, the accurate placement height of the Sapien 3 THV was checked by angiography. The Sapien 3 THV was inflated gradually to approach the aortic annulus. As soon as the frame of the Sapien 3 THV had touched the hinge points of the annulus, rapid pacing was discontinued accidentally. The Sapien 3 THV popped out into the ascending aorta, followed by immediate deflation of the deploying balloon (Figure 1). 


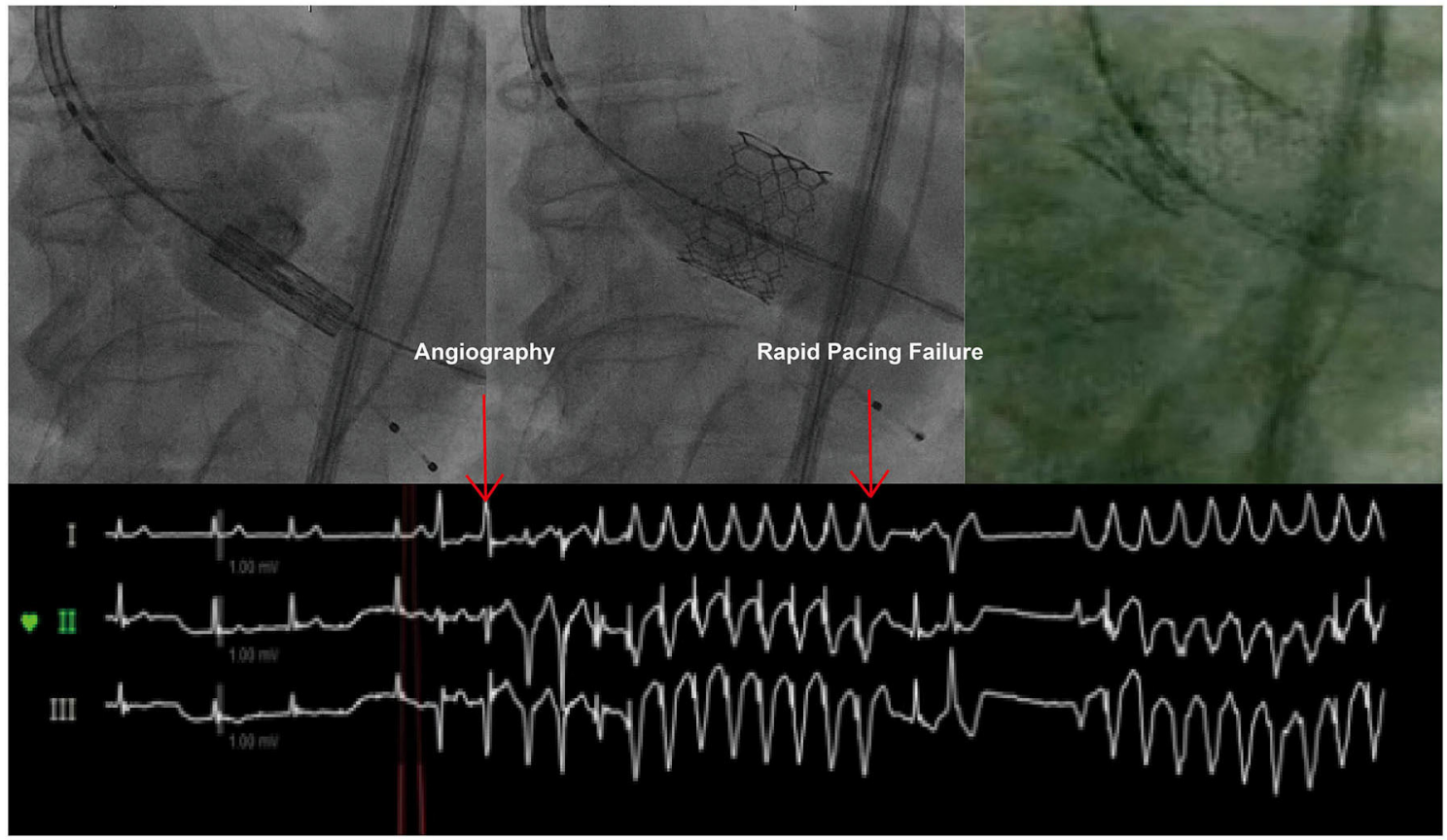

FIGURE 1 | Sapien 3 THV Embolization. Failure of rapid pacing caused Sapien 3 embolization.

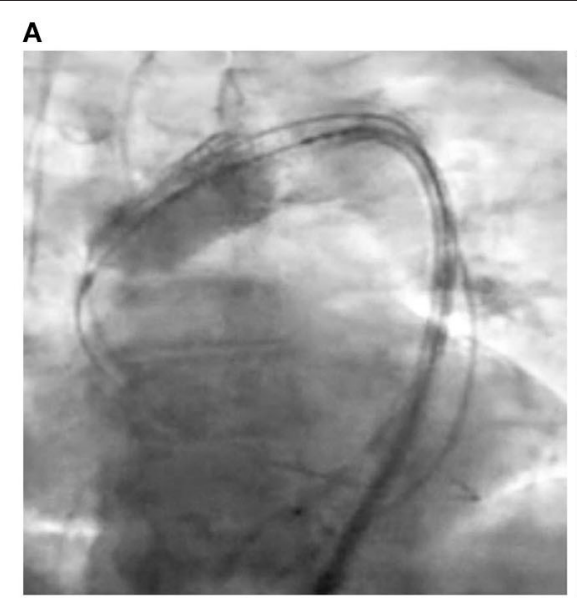

B

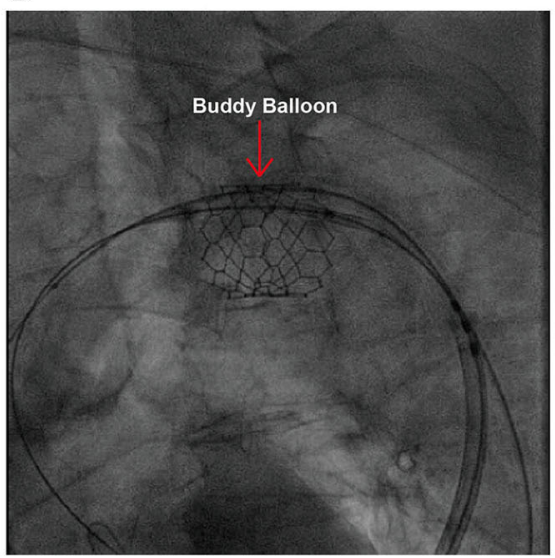

C

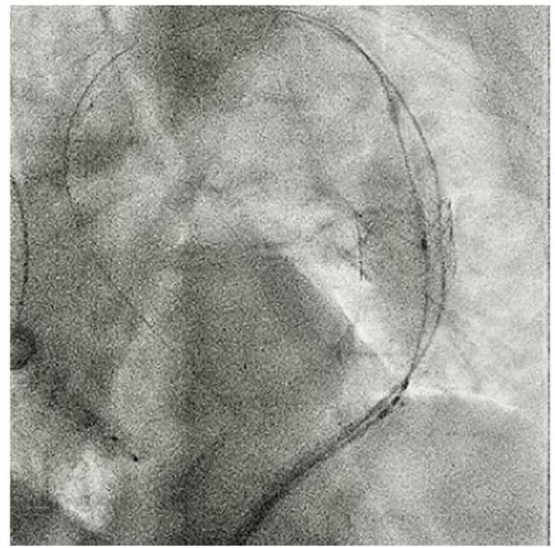

FIGURE 2 | Repositioning of the Embolized Sapien 3 THV. (A) Inflation of the deploying balloon to pull the whole system back. (B) Compression of the Sapien 3 THV by inflating a "buddy balloon." (C) Anchoring of the embolized Sapien 3 THV into the descending aorta.

Management of Sapien 3 THV Embolization First, management consisted of THV repositioning and anchoring, without causing any side branch obstruction. After keeping the stiff wire fixed in the left ventricle (LV) to avoid rotation of the THV, we inflated the deployment balloon and

Abbreviations: BEV, Balloon Expandable Valve; LV, Left Ventricle; TAVI, Transcatheter Aortic Valve Implantation; THV, Transcatheter Heart Valve. pulled it inside the inflow of the THV, thereby retrieving the whole system gently (Figure $\mathbf{2 A}$ ). The delivery system with the trapped THV was retracted smoothly but encountered resistance just before the origin of the left subclavian artery. We, therefore, attempted to crimp the Sapien 3 slightly. We used the pigtail catheter, pre-positioned for aortic root angiography, as a "buddy wire" to advance a $10 \times 40$ - $\mathrm{mm}$ balloon with a long J-wire (Figure 2B). A parallel access outside of the embolized THV in the arch was established to ensure the further manipulation. 

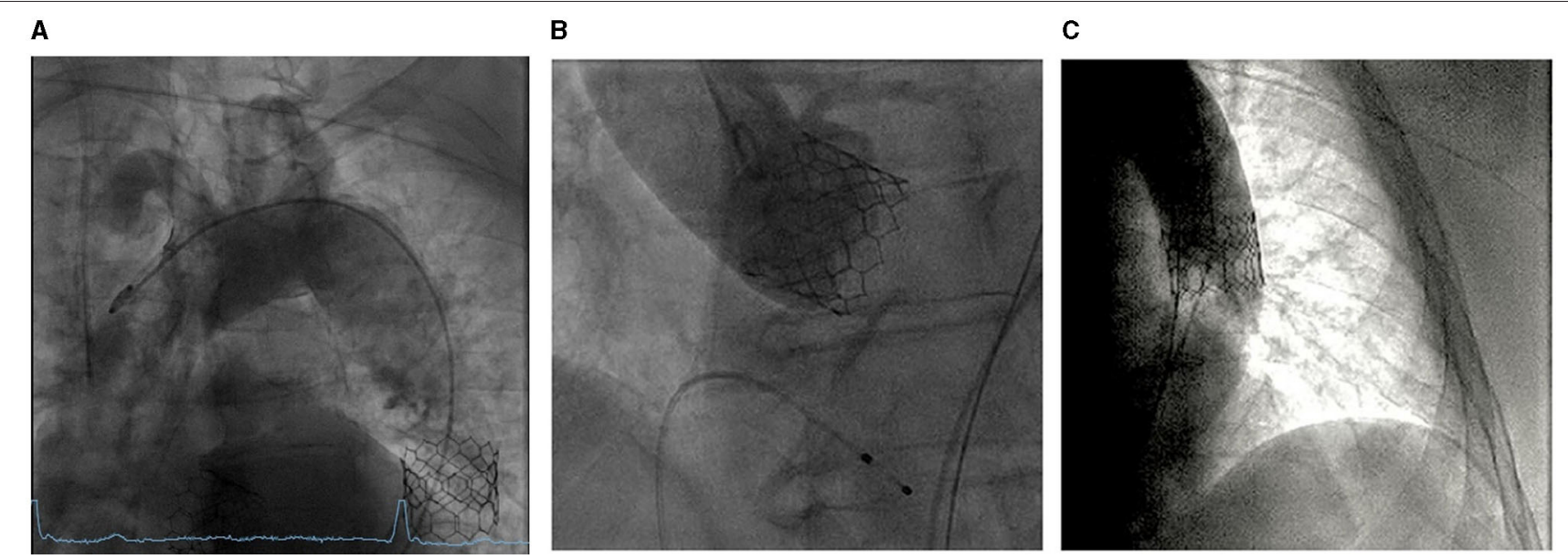

FIGURE 3 | Post-implantation evaluation. (A) Aortic root angiography for the evaluation of the second Sapien 3 THV. (B) Descending aorta angiography for the evaluation of the anchored Sapien 3 THV. (C) Aortic arch angiography for the exclusion of any occlusion of the main arteries.

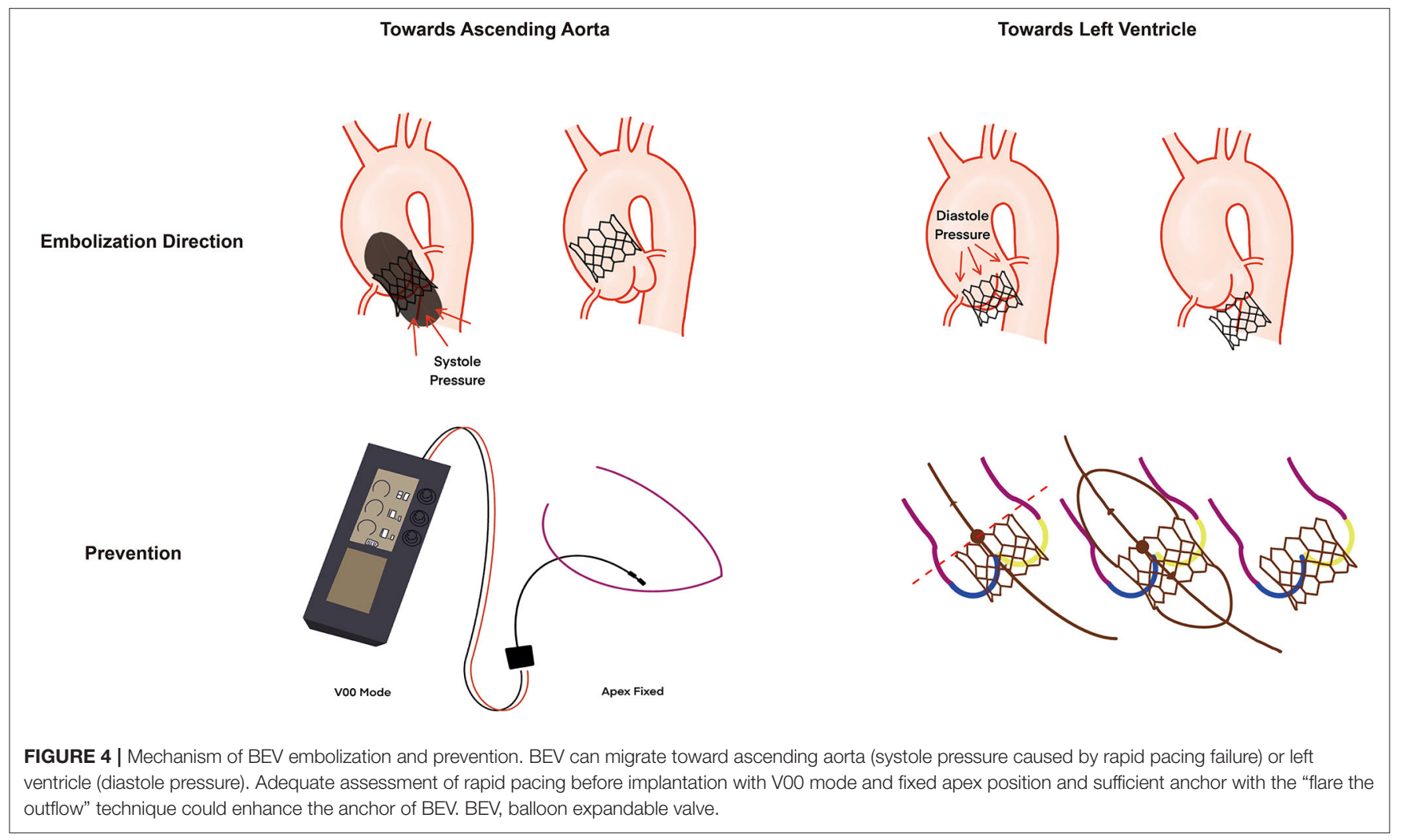

Having positioned the balloon adjacent to the Sapien 3 THV, the balloon was inflated and the Sapien 3 THV thereby compressed with counteractive force. The whole delivery system with the THV trapped by the deployment balloon could then be retrieved smoothly into the descending aorta.

The descending aorta was considered as an ideal anchoring position without origins of brachiocephalic arteries and other primary arteries. Sizing of the descending aorta was performed on the pre-TAVI CT. A location with a diameter of $28 \mathrm{~mm}$ was considered ideally to anchor without the further use of stenting. Then the Sapien 3 THV anchored in the descending aorta with $2 \mathrm{ml}$ overfilling of the deploying balloon (Figure 2C).

\section{Deployment of the Second Sapien 3 THV}

The second Sapien 3 THV was implanted and delivered through the first Sapien 3 THV. To avoid leaflet damage of the first 
Sapien 3 Embolization

Reposition and Anchor
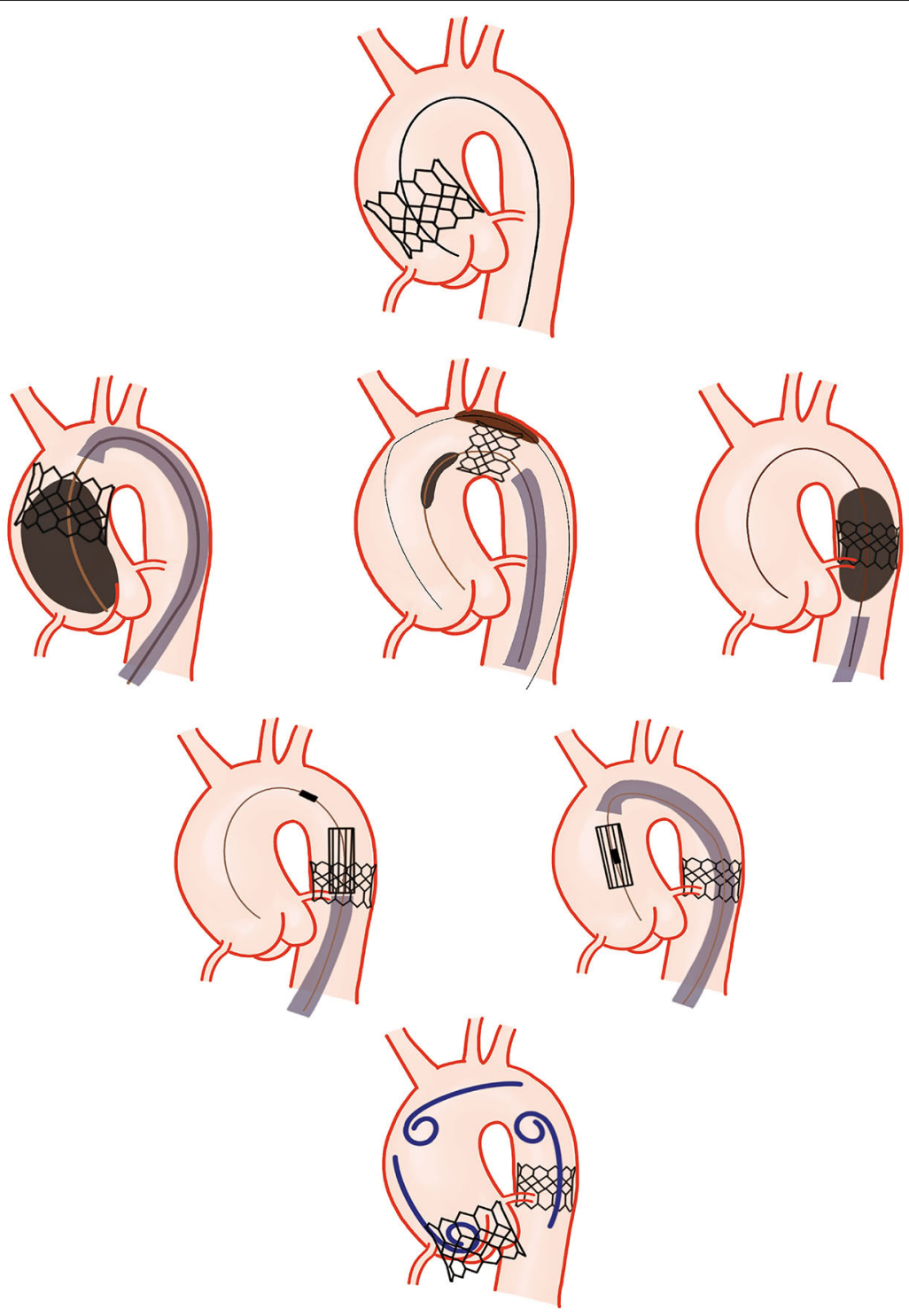

FIGURE 5 | Management of Sapien 3 THV Embolization. Schematic illustration of the management of a Sapien 3 THV embolization. First, repositioning and anchoring of the embolized Sapien 3 THV into the descending aorta with minimally invasive approaches are performed. Second, implantation of the second Sapien 3 THV with maximal centralization of the delivery system when crossing the embolized Sapien 3 THV. Third, angiography of the aortic root, the aortic arch, and the descending aorta is performed to evaluate THV function, patency of the brachiocephalic arteries, and the stability of the embolized valve in the descending aorta.

Sapien 3 THV, the delivery system was half-flexed to pass the first Sapien 3 THV centrally. Balloon alignment of the delivery system was commenced in the ascending aorta with adequate space but more resistance. The second Sapien 3 THV was deployed with $4 \mathrm{ml}$ overfilling, given that the annulus of $29.5 \mathrm{~mm}$ is outside of the recommended range $(2,3)$. "Flare the outflow" technique was performed at the height of the Sapien 3 outflow to achieve safe anchoring and preventing potential valve migration or embolization toward the left ventricle (LV) (4).

\section{Post-implantation Management}

Post-implantation management consisted of routine THV functional evaluation, ensuring anchoring of the embolized THV and excluding potential occlusion of main arteries. Invasive pressure assessment showed no transvalvular pressure gradient of the second THV. Aortic root angiography in functional projection showed an excellent result without any paravalvular leak (Figure 3A). The embolized Sapien 3 THV has inflated again with the second delivery system to ensure optimal anchoring. Angiography of the aortic arch and descending aorta was performed to confirm the optimal position of the second Sapien 3 THV and exclude any occlusion of the main arteries or migration of the embolized Sapien 3 THV (Figures 3B,C). The patient was under conscious sedation and in stable hemodynamic conditions throughout the procedure. The duration of the procedure was $85 \mathrm{~min}$. 


\section{DISCUSSION}

Transcatheter heart valve embolization is a rare but challenging complication of TAVI, with a reported incidence of about $1 \%$ (5). Rapid pacing failure remains one of the main individual causes of THV embolization, along with malpositioning, manipulation, and post-dilatation, with a higher prevalence in balloon-expandable valves (BEV) (5). Transcatheter heart valve embolization occurs toward the LV in case of insufficient anchoring of the valve (aortic pressure pushes the closed valve toward the left ventricle) or toward the aorta when rapid pacing during BEV deployment is interrupted (LV pressure pushes the valve toward the aorta) (Figure 4) (4). In this case, unexpected discontinuation of rapid pacing occurred without any obvious user error, which may be unavoidable. However, an adequate pre-implantation rapid pacing test should be confirmed carefully.

In this case, we summarized the management of an embolized balloon-expandable THV, including repositioning and anchoring of the embolized THV, implantation of the second THV, and post-implantation evaluation. Repositioning of the THV includes retraction of the valve on the balloon-shoulder with an inflated balloon, a snare, or a bioptome, which are, however, both associated with a substantial risk of aortic injury (6). As our first bailout strategy, the local deployment balloon was inflated again for retrieval with minimal invasiveness (embolized Sapien 3 THV is centered on the balloon-shoulder and not tilted). When the inflated THV was then stuck in the aortic arch, we used the second bailout strategy, consisting of a "buddy wire" and the advancement and inflation of a $10 \times 40-\mathrm{mm}$ balloon beside the embolized THV to compress the fully deployed valve and to bring it away from the outer curvature of the aortic arch and the outgoing arteries.

When embolization has occurred, a well-cooperating Heart Team is crucial to successfully tackle the complication. Overall management is performed by the operators (Figure 5, Supplementary Video 1). The scrub nurse is responsible to prepare and crimp the second THV immediately, and anesthesiologists are in charge of maintaining stable hemodynamics. Mechanical circulation support should be on standby when it is necessary.

\section{FOLLOW-UP}

After the procedure, the patient was sent to the intermediate care unit. He was conscious without neurologic symptoms.

\section{REFERENCES}

1. Writing Committee Members, Otto CM, Nishimura RA, Bonow RO, Carabello BA, Erwin JP III, et al. 2020 ACC/AHA guideline for the management of patients with valvular heart disease: a report of the American College of Cardiology/American Heart Association Joint Committee on clinical practice guidelines. J Am Coll Cardiol. (2021) 77:e25-197. doi: 10.1016/j.jacc.2020.11.018

2. Shivaraju A, Kodali S, Thilo C, Ott I, Schunkert H, von Scheidt W, et al. Overexpansion of the SAPIEN 3 transcatheter heart valve: a feasibility
Electrocardiography showed atrial fibrillation with bradycardia. Laboratory tests had no positive findings. The preexisting anticoagulation with a non-vitamin $\mathrm{K}$ antagonist oral anticoagulant (NOAC) was continued $6 \mathrm{~h}$ after the intervention.

\section{CONCLUSIONS}

Failure of rapid pacing may cause THV embolization. Overall management of this complication should include repositioning the first THV, modified implantation of the second THV, and an adequate THV evaluation after the second implantation.

\section{LEARNING OBJECTIVES}

- Failure of rapid pacing could cause THV embolization toward the ascending aorta.

- Embolization management includes first THV repositioning, second THV implantation, and careful post-implantation evaluation.

- The Sapien 3 THV could be compressed and navigated successfully through the aortic arch by using a "buddy balloon" and performing a pullback maneuver into the descending aorta on the shoulder of the inflated deployment balloon.

\section{DATA AVAILABILITY STATEMENT}

The raw data supporting the conclusions of this article will be made available by the authors, without undue reservation.

\section{AUTHOR CONTRIBUTIONS}

$\mathrm{MC}$ and $\mathrm{AK}$ conceived of the presented idea of the paper. MC wrote the manuscript with support from AK, BS, JM, $\mathrm{MB}$, and FT. MC drew the figures. AK supervised the project. All authors contributed to the article and approved the submitted version.

\section{SUPPLEMENTARY MATERIAL}

The Supplementary Material for this article can be found online at: https://www.frontiersin.org/articles/10.3389/fcvm. 2021.774047/full\#supplementary-material

Supplementary Video 1 | Embolized Sapien 3 THV: Cause, management, and redo. THV, transcatheter heart valve. 
5. Kim WK, Schafer U, Tchetche D, Nef H, Arnold M, Avanzas P, et al. Incidence and outcome of peri-procedural transcatheter heart valve embolization and migration: the TRAVEL registry (TranscatheteR HeArt Valve EmboLization and Migration). Eur Heart J. (2019) 40:3156-65. doi: 10.1093/eurheartj/ehz429

6. Alkhouli M, Sievert H, Rihal CS. Device embolization in structural heart interventions: incidence, outcomes, and retrieval techniques. JACC Cardiovasc Interv. (2019) 12:113-26. doi: 10.1016/j.jcin.2018.08.033

Conflict of Interest: AK is a consultant and proctor for Edwards Lifesciences.

The remaining authors declare that the research was conducted in the absence of any commercial or financial relationships that could be construed as a potential conflict of interest.
Publisher's Note: All claims expressed in this article are solely those of the authors and do not necessarily represent those of their affiliated organizations, or those of the publisher, the editors and the reviewers. Any product that may be evaluated in this article, or claim that may be made by its manufacturer, is not guaranteed or endorsed by the publisher.

Copyright (c) 2021 Chen, Stähli, Michel, Brinkert, Tanner and Kasel. This is an open-access article distributed under the terms of the Creative Commons Attribution License (CC BY). The use, distribution or reproduction in other forums is permitted, provided the original author(s) and the copyright owner(s) are credited and that the original publication in this journal is cited, in accordance with accepted academic practice. No use, distribution or reproduction is permitted which does not comply with these terms. 